\title{
Acúmulo de Açucares em Variedades de Cana Influenciadas por Diferentes supressões de Irrigação e Adubação
}

\author{
Franklin Meireles de Oliveira *, Ignacio Aspiazú, Marcos Koiti Kondo, Iran Dias Borges, Rodinei Facco Pegoraro, \\ Poliana Batista de Aguilar \\ *Autor para correspondência \\ Parte da dissertação de Mestrado do primeiro autor, apresentada ao Programa de Pós-Graduação em Produção \\ Vegetal no Semiárido do Instituto de Ciências Agrárias da Universidade Estadual de Montes Claros, \\ Av. Reinaldo Viana, 2.630, C. P. 91, Janaúba-MG, Brasil, 39.440-000. \\ Grupo do Departamento de Ciências Agrárias/ Manejo de grandes culturas, CCET-UNIMONTES-MG, \\ correspondente: franklin.meireles@yahoo.com.br \\ endereço: Rua Grão Mogol, 474, Vila Formosa, Taiobeiras-MG, 39550-000.
}

\section{RESUMO}

Objetivou-se, neste trabalho, avaliar as características tecnológicas de acúmulo de açúcares e ação da invertase de duas variedades de cana-de-açúcar submetidas a diferentes supressões de irrigação e níveis de adubação. O experimento foi instalado na Fazenda Experimental da Universidade Estadual de Montes Claros, em Janaúba-MG. O solo da área experimental foi classificado como Latossolo Vermelho Distrófico textura média, (LVd); (EMBRAPA, 1999), o delineamento utilizado foi o de blocos casualizados, três repetições, em parcelas subdivididas $(2 \times 3 \times 6)$, sendo duas variedades de cana-de-açúcar RB85- 5453 e SP80-1816, três épocas de supressão de irrigação mais precipitação após corte (DIAC) caracterizadas aos 165, 195 e 225 dias, e na subparcela adubouse seis níveis de adubação mineral. As variáveis foram: brix, AR, ART, ATR e umidade. Os níveis de adubação interferiram na qualidade do caldo obtendo uma média geral de $24,30^{\circ}$ brix, com a variedade RB85-5453 obtendo maiores valores para todas as variáveis analisadas. Observou-se valor de 0,8\% AR na variedade SP80-1816 e mínimo na variedade RB85-5453 com 0,35 \%AR, no parâmetro ATR, verificou-se que a variedade RB85-5453 apresentou melhor resultado, tendo possibilidade de atingir teores superiores a 189,25 kg de ATR t-1. O nível 6 de adubação obteve melhor desempenho nas condições de realização deste trabalho. A RB85-5453 é a mais indicada para a região, pois conseguiu altos níveis em produção industrial.

PALAVRAS CHAVE: análises industriais, fertilização, semiárido e sacarose.

\section{Accumulation of Sugars in Sugarcane Varieties Affected by Deletions of Different Irrigation and Fertilization}

\begin{abstract}
The aim of this study was to evaluate the technical characteristics of the accumulation of sugars and invertase action of two varieties of sugar cane under different deletions of irrigation and fertilization levels. The experiment was conducted at the Experimental Farm of Universidade Estadual de Montes Claros, in Janaúba-MG. The experimental area was classified as Oxisol, medium texture, (Oxisol) (EMBRAPA, 1999), the design was a randomized complete block, three replications in split plot $(2 \times 3 \times 6)$, two varieties of cane sugar RB85-5453 and SP80-1816, three times more precipitation suppression irrigation after cutting (DIAC) characterized at 165, 195 and 225 days, and fertilizer as subplots were six levels of mineral fertilizer. The variables were: brix, AR, TRS, TRS, and humidity. Nutrient levels interfere with the quality of the juice obtained an overall average of $24.30^{\circ}$ brix, with the variety RB85-5453 obtaining higher values for all variables. Observed value of $0.8 \%$ AR in the variety SP80-1816 and minimum in variety RB85-5453 with $0.35 \%$ AR in the ATR parameter, it was found that the variety RB85-5453 showed better results, with the possibility of reaching levels higher than $189.25 \mathrm{~kg}$ TRS t-1. 6 The level of fertilization showed the best performance in terms of this work The RB85-5453 is the best for the region because high levels achieved in industrial production.
\end{abstract}

KEYWORDS: industrial analysis, fertilization, semiarid and sucrose. 


\section{INTRODUÇÃO}

Em todo o Brasil, a cana-de-açúcar tem sido remunerada por seus índices qualitativos, de modo que, quanto melhor a qualidade da matéria prima maior é o preço pago por tonelada de colmos. A cultura da canade-açúcar passou a ser considerada, recentemente, uma realidade no quesito energético, valorizada pela capacidade que tem de gerar energia limpa e renovável. Em nível mundial, começa a haver uma corrida por combustíveis mais 'limpos', entretanto, quando o assunto é expandir as fronteiras agrícolas para a produção de bicombustíveis, teme-se os seus reflexos na diminuição de áreas para a produção de alimentos. Essa parece ser uma das grandes vantagens para o Brasil, país de dimensões continentais, dispondo, ainda, de terras para a expansão de cultivo da cana-de-açúcar, além de renovação na extração de etanol uma sustentável fonte de energia combustível.

Diversos fatores interferem na produção e maturação da cultura da cana-de-açúcar, sendo os principais a interação edafoclimática, o manejo da cultura e a cultivar escolhida (César et al., 1987). Esses fatores que interferem na produção e qualidade da cana-de-açúcar estão sendo constantemente estudados sob diferentes aspectos. Estudar a cultura no seu ambiente de desenvolvimento pode gerar uma enorme quantidade de informações para adequar o melhor manejo e cultivar para os específicos ambientes (solo e clima). Assim é possível explorar ao máximo o local de produção para conseqüentemente maior lucratividade ou competitividade para as agroindústrias da cana-de- açúcar.

A refratometria, na escala brix, constitui em um método físico para medir a quantidade de sólidos solúveis presentes em uma amostra. Baseia-se em um sistema de graduação de aparelhos especialmente para ser utilizado na indústria açucareira, mais precisamente na análise de açúcares em geral que estejam em solução. Segundo Delgado \& César (1977); Paranhos (1987); Lopes \& Parazzi (1992) a maturação da cana-de-açúcar pode ser determinada pelos parâmetros tecnológicos (Brix, Pol, Pureza e Açúcares Redutores).

Segundo Horii (2004) as cultivares de cana-deaçúcar, devido aos diferentes comportamentos de maturação, são agrupadas em precoces (quando apresentam um teor de Pol acima de $13 \%$ no início de maio), médias (quando atingem a maturação em julho) e tardias (pico de maturação em agosto/setembro). Além disto podem ser consideradas como de PUI curto (< 120 dias), PUI médio (120 a 150 dias) e PUI longo (>150 dias).

Em algumas regiões do País, a exemplo do Nordeste e, especificamente, no Norte de Minas Gerais, há interesses em se verticalizar a produção, aumentando a produtividade agrícola e industrial, com melhorias na qualidade da matéria-prima. Nesse sentido, os produtores Mineiros têm investido, maciçamente, em irrigação e adubação, como principais tecnologias para o aumento de produtividade e qualidade da cultura de cana-deaçúcar. Considerando a escassez de recursos hídricos, um dos problemas da expansão da agricultura irrigada no Estado, inclusive no setor sucroalcooleiro, é imprescindível e urgente se estudar práticas de manejo mais eficientes do uso de água, igualmente importante é se identificar os principais problemas de ordem nutricional das plantas, em cada área de produção, visando garantir a sustentabilidade da exploração da cultura.

$\mathrm{Na}$ literatura, são variadas as informações sobre demanda hídrica da cana-de-açúcar, segundo Doorembos \& Kassam (1979), a cultura exige entre 1500 e $2500 \mathrm{~mm}$ ano-1. Estudando os efeitos da irrigação sobre a qualidade da cana 'CP 65-357, submetida a três níveis de irrigação (99, 85 e 65\% da fração de esgotamento da água do solo), no Estado do Texas, Wiedenfeld (1995) obteve rendimentos em açúcar de 13, 10 e 7,5 t ha-1, respectivamente, sem variar a pureza (87, 86 e $85 \%$ ), posteriormente, Wiedenfeld (2000) avaliou diferentes tratamentos de estresse hídrico na cultura, em cinco diferentes estádios de desenvolvimento, registrando reduções de $11 \%$ no rendimento em açúcar, quando a cultura foi submetida ao estresse no período entre 257 e 272 dias após o plantio e de $19 \%$ quando o estresse hídrico ocorreu entre 302 e 347 dias após o plantio. Dantas Neto et al. (2006) testaram lâminas de irrigação, variando entre 807 e 1343 mm, na cultura de cana-de-açúcar e verificaram comportamento linear positivo sobre as variáveis de crescimento e quadrático para sacarose (POL), com valor de $18,1 \%$ quando as plantas foram irrigadas com $1125 \mathrm{~mm}$. Moura et al. (2005) observaram diferenças em ${ }^{\circ}$ Brix, Pol e PCC em estudos, comparando a cana irrigada com a cana de sequeiro, aumentando 5,00, 10,79 e $8,63 \%$, respectivamente, quando houve irrigação.

Dentro deste enfoque, procurando atender às necessidades atuais de manejo, o presente trabalho teve por objetivo estudar o comportamento de duas variedades comerciais de cana-de-açúcar, quanto às características tecnológicas de acúmulo de açúcares e ação da invertase influenciada por diferentes épocas de supressão de irrigação e distintos níveis de adubação, nas condições edafoclimáticas da região Norte do Estado de Minas Gerais.

\section{MATERIAL E MÉTODOS}

O experimento foi instalado na Fazenda Experimental da Universidade Estadual de Montes Claros, em Janaúba - MG. O solo da área experimental foi classificado como Latossolo Vermelho Distrófico textura média, (LVd); (EMBRAPA, 1999), o delineamento utilizado foi o de blocos casualizados, com três repetições, em parcelas subdivididas $(2 \times 3 \times 6)$, sendo utilizado na parcela duas variedades de cana-de-açúcar RB855453 e SP80-1816 e três épocas distintas de supressão de irrigação mais precipitação após corte (DIAC) 
caracterizadas aos 165, 195 e 225 dias, foi utilizado o sistema de irrigação por aspersão convencional com aspersores de baixa pressão. A lâmina de irrigação foi determinada levando-se em consideração a precipitação e eficiência do sistema utilizado, bem como a evapotranspiração da cultura (ETc e Kc da cana-de-açúcar) (BERNARDO et al., 2005), dispensando-a na ocorrência de precipitação. Dados climáticos obtidos a partir da estação meteorológica da Epamig, Nova Porteirinha-MG. Na subparcela adubou-se em uma só vez seis níveis de adubação mineral, 1 (0 kg ha-1 de N e K2O), 2 (14 kg ha-1 de N e 33 kg ha-1 de K2O), 3 (29 kg ha-1 de N e $66 \mathrm{~kg}$ ha-1 de K2O), 4 (43 kg ha-1 de N e $100 \mathrm{~kg}$ ha-1 de K2O), 5 (57 kg ha-1 de N e 133 kg ha-1 de K2O), 6 (71 kg ha-1 de N e $166 \mathrm{~kg}$ ha-1 de K2O) das fontes de Sulfato de Amônia e Cloreto de Potássio. As variáveis da cultura utilizadas foram: brix, AR, ART, ATR e umidade. Foram feitas duas analises de todas as parcelas, a primeira analise no mês de agosto (300 dias após corte) e a segunda em setembro (330 dias após corte) de 2010 no laboratório Industrial da Usina São Judas Tadeu (SADA Bioenergia e Agricultura), no município de Jaíba-MG, foram coletados 3 colmos inteiros aleatórios em cada sub-parcela e transportados no transcorrer de 1 hora para o laboratório, simulando o tempo real de coIheita, conforme (CONSECANA, 2006).

Análise de Brix e Pol foram feitos pelo método da prensa hidráulica, sendo do caldo feita a análise do Brix (refratométrico) e determinada por métodos sacarimétricos (polarímetros ou sacarímetros) o Pol\% caldo = $(1,0078 \times$ leit. sacar. $+0,0444) \times(0,2607-0,009882 \times$ Brix). O bagaço foi chamado de "Bolo Úmido". O peso do bolo úmido (P.B.U.) foi obtido em balança eletrônica. Os resultados obtidos (leitura Sacarimétrica, Brix e
PBU), foram lançados em um programa de computador (CHB), obtendo-se os demais resultados: Pol da cana, fibra, pureza, AR, brix da cana, umidade, ATR e ART com os seguintes cálculos: Fibra $=(0,08 \times \mathrm{PBU})+0,876$ (FERNANDES, 2000). O coeficiente " $C$ " representa a transformação do caldo extraído em todo o caldo absoluto, ou seja, é a extração de todo caldo proveniente da prensa hidráulica. $C=(1,0313-0,00575 \times$ FIBRA $)$. Posteriormente encontra-se o Brix \% cana $=$ Brix do caldo $\mathrm{x}$ $(1-0,01) \times C$ e Pol\% cana $=$ Pol no caldo $\times(1-0,01 \times$ FIBRA) $x$ C, a pureza foi obtida pela fórmula: PUREZA = (Pol\% cana) / (Brix\% cana) x 100. O cálculo dos açúcares redutores no caldo foi feito pela fórmula: $A R \%$ caldo $=(3,641-0,0343 \times$ PUREZA $) ;$ a AR\% cana $=$ AR no caldo $\times(1-0,01 \times$ FIBRA $) \times C$. Os açúcares redutores totais foram determinados pela seguinte equação: ART\% caldo $=($ Pol no caldo $/ 0,95)+$ AR e ART $\%$ cana $=$ ART do caldo x (1-0,01 x FIBRA) x C. Conhecendo-se a Pol da cana (PC) e os açúcares redutores da cana (ARC), o açúcar total recuperável é calculado pela equação: ATR $=10 \times$ PC x 1,05263 x 0,905 + $10 \times$ ARC x 0,905, onde: $10 \times \mathrm{PC}=$ pol por tonelada de cana; $1,05263=$ coeficiente estequiométrico para a conversão da sacarose em açúcares redutores; 0,905 = coeficiente de recuperação, para uma perda industrial de $9,5 \%$ (nove e meio por cento) e $10 \times$ ARC = açúcares redutores por tonelada de cana. O Álcool hidratado estimado pelo calculo: ART\% caldo $\times 10 \times 0,6475=100 \%$ de álcool, posteriormente transforma-se para $85 \%$, considerada a eficiência do processo fermentativo.

A distribuição da precipitação no experimento ao longo do ciclo fenológico da cultura (360 dias), em intervalos quinzenais encontra-se na Figura 1.

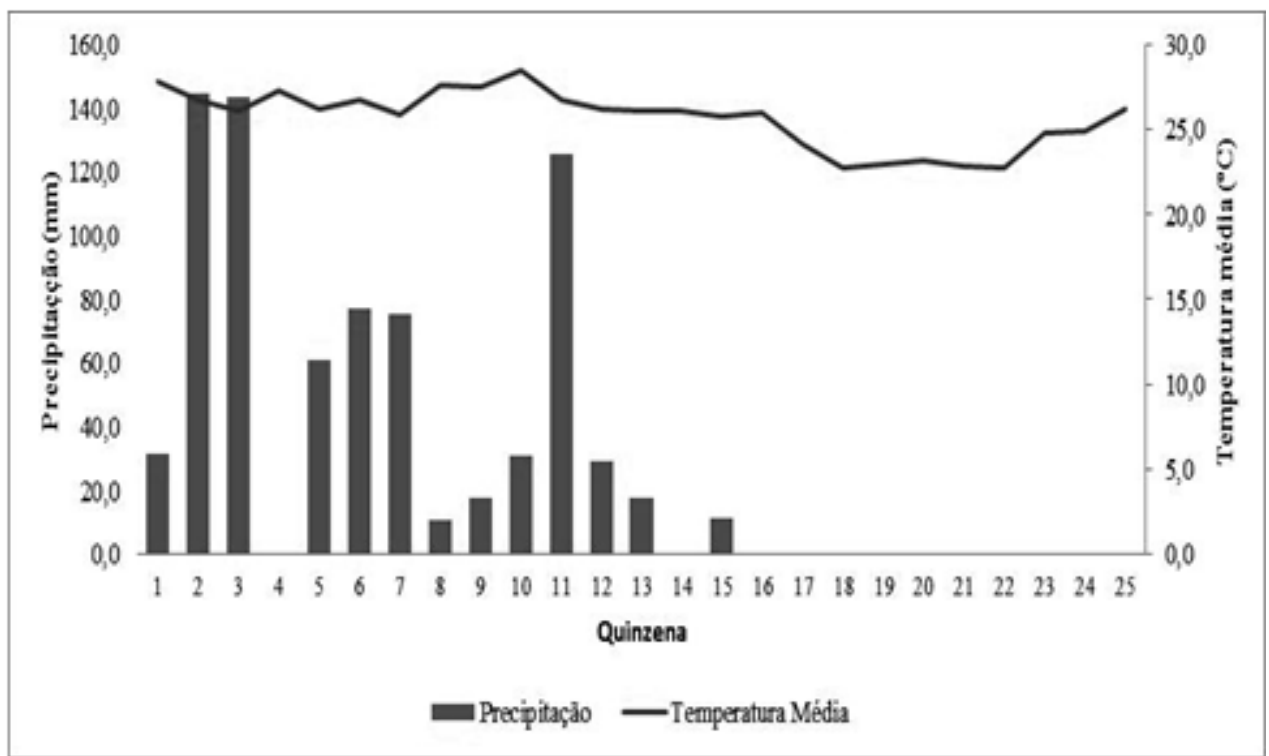

Figura 1. Dados médios de temperatura, em graus Celsius $\left({ }^{\circ} \mathrm{C}\right)$, e precipitação pluvial acumulada por quinzena, em milímetros (mm), em Janaúba-MG, de 01/10/2009 a 30/09/2010. Dados obtidos na Estação Climatológica da EPAMIG, Nova Porteirinha-MG, 2011. 
As quantidades de água aplicadas por meio da irrigação durante os três períodos de supressão foram, L1 $(165$ DIAC $)=918 \mathrm{~mm}$, L2 $(195$ DIAC $)=1.242 \mathrm{~mm}$ e L3 $(225 \mathrm{DIAC})=1.539 \mathrm{~mm}$. A precipitação aproveitável (Pap) acumulada no mesmo período foi de 873,8 ; 891,2; e $902,4 \mathrm{~mm}$ respectivamente, resultando em quantidades totais de água aplicadas para os três DIAC distintos de L1 + Pap ( 165 DIAC) $=1.791,8 \mathrm{~mm}, \mathrm{~L} 2+$ Pap $(195$ $D I A C)=2.133,2 \mathrm{~mm}$ e L3 + Pap $(225 \mathrm{DIAC})=2.441,4$ $\mathrm{mm}$.

Foi feita a média dos dados de coleta dos dois meses, esta media foi submetida à análise de variância e, quando houve diferença significativa pelo teste $F$, realizou-se a comparação das medias pelo teste de Scott Knott $(p<0,05)$ para todas as variáveis estudadas, utilizando-se do programa software SISVAR.

\section{RESULTADOS E DISCUSSÃO}

Verifica-se na tabela 1 que há interações entre variedades, DIAC e níveis de adubação, tendo a variedade RB85-5453 valores maiores para todas as variáveis analisadas, o que pode ser explicado pelo seu comportamento precoce em sua maturação.

Tabela 1. Resumo das análises de variância para variáveis Umidade, Brix, Açúcares teórico recuperáveis (ATR), açúcares redutores (AR) e açucares redutores totais (ART), Janaúba, MG, 2010.

\begin{tabular}{|c|c|c|c|c|c|c|}
\hline \multicolumn{7}{|c|}{ QUADRADOO MÉDIO } \\
\hline F.V. & G.L & $\begin{array}{c}\text { UMIDADE } \\
\text { (\%) }\end{array}$ & $\begin{array}{l}\text { BRIX } \\
(\%)\end{array}$ & $\begin{array}{c}\text { ATR } \\
\mathrm{Kg} \mathrm{t}^{-1}\end{array}$ & ART & $\begin{array}{l}\text { AR } \\
(\%)\end{array}$ \\
\hline BLOCO & 2 & 1,7759 & 0,560 & 7,32 & 0.3719 & 0,0004 \\
\hline VAR & 1 & $241,8014^{*}$ & $150,92^{*}$ & $10583,892^{*}$ & $136.5075^{*}$ & $0,430^{*}$ \\
\hline DIAC & 2 & $11,0759^{*}$ & $7,47^{*}$ & $419,7014^{*}$ & 4.7971 & $0,016^{*}$ \\
\hline VAR*DIAC & 2 & 2,8071 & $3,51^{*}$ & $286,7941^{*}$ & 4.4560 & $0,027^{*}$ \\
\hline erro 1 & 10 & 1,9244 & 0,795 & 69,027 & 1.2784 & 0,0001 \\
\hline Nív & 5 & 1,1792 & 0,706 & 78,237 & 1.4494 & $0,026^{*}$ \\
\hline VAR*NÍV & 5 & 0,6431 & 0,301 & 44,747 & 0.5487 & $0,010^{*}$ \\
\hline DIAC*NÍV & 10 & $1,6670^{*}$ & 0,766 & 74,439 & 0.8988 & $0,018^{*}$ \\
\hline $\begin{array}{c}\text { VAR*DIAC* } \\
\text { NÍV }\end{array}$ & 10 & $0,8145^{*}$ & 0,564 & 40,435 & 0.4561 & $0,004^{*}$ \\
\hline erro 2 & 60 & 0,7356 & 0,408 & 52,724 & 0.7683 & 0,0009 \\
\hline $\begin{array}{l}\text { Total corri- } \\
\text { gido }\end{array}$ & 107 & & & & & \\
\hline CV $1(\%)=$ & 2 & 2,09 & 3,67 & 4,82 & 5.96 & 4,33 \\
\hline CV $2(\%)=$ & 1 & 1,29 & 2,63 & 4,21 & 4.62 & 5,14 \\
\hline Média geral: & 2 & 66,496 & 24,308 & 172,388 & 18.986 & 0,598 \\
\hline
\end{tabular}

1 variedade (VAR), 2 período em dias de precipitação mais irrigação até a época de supressão (DIAC), 3 níveis de adubação (NIV). * valores significativos estatisticamente $(p<0,05)$ pelo teste $F$.

As variedades apresentaram uma média geral de $24,30^{\circ}$ Brix (Tabela 1). Os atributos de qualidade da matéria prima são os mais importantes para a indústria canavieira, visto que vão definir os rendimentos em açúcar e álcool.

Constata-se na tabela 2 que as variedades diferiram estatisticamente na variável Brix em todos os tratamentos, com a variedade RB85-5453 superando em valores a variedade SP80-1816. Nos tratamentos da variedade RB85-5453 não houve diferenças entre DIAC, logo as diferentes supressões de irrigação não interferiram na porcentagem de sólidos solúveis acumulados na variedade, porém, na SP80-1816 houve diferenças entre os DIAC dentro de cada nível de adubação com os menores valores encontrados nos menores DIAC. A água disponível para a planta é essencial, uma vez que promove a síntese, acúmulo e a translocação da sacarose nos colmos, podendo os produtores manipular a irrigação visando à melhoria da maturação. As diferentes disponibilidades hídricas e nutricionais não afetaram a umidade das variedades estudadas. Relatou-se valor máximo de $70 \%$ aos 165 DIAC no nível 2 na variedade SP80-1816 e mínimo de 64,36 \% no nível 1 aos 195 DIAC da variedade RB85-5453. 
TABELA 2 Médias dos valores de Brix em caldo e umidade no desdobramento do período de irrigação e precipitação (DIAC) com variedades dentro de cada nível de adubação.

\begin{tabular}{|c|c|c|c|c|c|}
\hline \multirow[b]{2}{*}{ NÍVEIS } & \multirow[b]{2}{*}{ DIAC } & \multicolumn{2}{|c|}{$\begin{array}{c}\text { BRIX } \\
(\%)\end{array}$} & \multicolumn{2}{|c|}{$\begin{array}{c}\text { UMIDADE } \\
(\%)\end{array}$} \\
\hline & & SP80-1816 & RB85-5453 & SP80-1816 & RB85-5453 \\
\hline \multirow{3}{*}{1} & 165 & $21,89 \mathrm{bA}$ & $24,74 a A$ & $68,28 a A$ & $64,70 \mathrm{bA}$ \\
\hline & 195 & $21,41 \mathrm{bA}$ & $24,84 \mathrm{aA}$ & $68,10 \mathrm{aA}$ & $64,36 \mathrm{bA}$ \\
\hline & 225 & $22,79 \mathrm{bA}$ & $23,81 \mathrm{aA}$ & $67,41 \mathrm{aA}$ & $64,98 \mathrm{bA}$ \\
\hline \multirow{3}{*}{2} & 165 & $21,85 \mathrm{bB}$ & $25,36 a A$ & $70,00 a A$ & 65,46 bA \\
\hline & 195 & $23,35 \mathrm{bA}$ & $25,33 a A$ & $67,55 \mathrm{aB}$ & $64,88 \mathrm{bA}$ \\
\hline & 225 & $23,57 \mathrm{bA}$ & $25,08 \mathrm{aA}$ & $67,66 \mathrm{aB}$ & $65,81 \mathrm{bA}$ \\
\hline \multirow{3}{*}{3} & 165 & $22,58 \mathrm{bB}$ & $25,22 \mathrm{aA}$ & $68,56 \mathrm{aA}$ & $65,45 \mathrm{bA}$ \\
\hline & 195 & $22,38 \mathrm{bB}$ & $24,97 \mathrm{aA}$ & $68,81 \mathrm{aA}$ & $65,28 \mathrm{bA}$ \\
\hline & 225 & $24,03 \mathrm{bA}$ & $25,71 \mathrm{aA}$ & $67,18 a A$ & $64,61 \mathrm{bA}$ \\
\hline \multirow{3}{*}{4} & 165 & $21,97 \mathrm{bB}$ & $25,24 a A$ & $69,10 a A$ & $65,63 \mathrm{bA}$ \\
\hline & 195 & $23,25 \mathrm{bA}$ & $24,57 a A$ & $67,68 \mathrm{aA}$ & $66,00 \mathrm{bA}$ \\
\hline & 225 & $24,39 \mathrm{bA}$ & $25,55 \mathrm{aA}$ & $66,26 \mathrm{aB}$ & 64,68 aA \\
\hline \multirow{3}{*}{5} & 165 & $23,36 \mathrm{bA}$ & $25,68 \mathrm{aA}$ & $67,96 \mathrm{aA}$ & $65,06 \mathrm{bA}$ \\
\hline & 195 & $21,87 \mathrm{bB}$ & $25,45 a A$ & 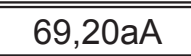 & $\begin{array}{c}65,35 \mathrm{bA} \\
\end{array}$ \\
\hline & 225 & $24,07 \mathrm{bA}$ & $26,25 a A$ & $66,73 \mathrm{aB}$ & $63,80 \mathrm{bA}$ \\
\hline \multirow{3}{*}{6} & 165 & $22,55 \mathrm{bA}$ & $26,01 \mathrm{aA}$ & $68,38 \mathrm{aA}$ & $64,63 \mathrm{bA}$ \\
\hline & 195 & $23,06 \mathrm{bA}$ & $25,10 a A$ & $67,65 \mathrm{aA}$ & $65,30 \mathrm{bA}$ \\
\hline & 225 & $23,85 \mathrm{bA}$ & $25,87 a A$ & $67,30 a A$ & $64,01 \mathrm{bA}$ \\
\hline
\end{tabular}

(1)Nível 1 (0 kg ha-1 de N e K2O), 2 (14 kg ha-1 de N e 33 kg ha-1 de K2O), 3 (29 kg ha-1 de N e 66 kg ha-1 de K2O), 4 (43 kg ha-1 de N e 100 kg ha-1 de K2O), 5 (57 kg ha-1 de N e 133 kg ha-1 de K2O), 6 (71 kg ha-1 de N e $166 \mathrm{~kg}$ ha-1 de K2O). (2)Médias dentro de cada atributo seguidas por letras minúsculas distintas na linha diferem entre si estatisticamente pelo teste de Scott Knott a $5 \%$ de probabilidade. (3)Médias dentro de cada nível seguidas por letras maiúsculas distintas na coluna diferem entre si estatisticamente pelo teste de Scott Knott a $5 \%$ de probabilidade.

Melo et al. (1998) verificaram, para diferentes variedades, média de umidade de $70,64 \%$ para cana integral e $71,88 \%$ para cana despontada, ratificando os valores encontrados neste trabalho.

$\mathrm{Na}$ tabela 3 encontram-se as médias de níveis de adubação dentro de cada DIAC e variedades. Para a variável Brix, houve diferença significativa apenas aos 195 DIAC na variedade SP80-1816. Algumas variedades têm comportamento distinto com relação ao nitrogênio sendo capazes de utilizar mais nitrogênio do que outras, como também em relação o acúmulo de sacarose, ou seja, altas doses podem não afetar a qualidade dos caldos. É um efeito variável, que tem sido observado. Vários trabalhos demonstraram que água e nitrogênio estando em excesso, a planta não amadurece, correlacionando-se positivamente com a umidade e açúcares redutores, e negativamente com a sacarose.

Nesta pesquisa, as variedades mostraram comportamento oposto, o nitrogênio não interferiu na quali- dade do caldo pelo fato, principalmente, da quantidade disponibilizada dos diferentes níveis de adubação nitrogenada que se encontraram de moderado para baixo de acordo com CFSEMG (1999). No caso de cana irrigada, a maturação deve ser monitorada mediante o controle da aplicação de nitrogênio e da irrigação. A redução da água para controle da irrigação diminui a absorção de nitrogênio pela planta. Em regiões úmidas e quentes, é difícil reduzir a quantidade de água do solo nos períodos de maturação e, consequentemente, a quantidade de nitrogênio aplicada deve ser moderada.

O potássio favoreceu a síntese, o acúmulo e a translocação da sacarose, aumentando a qualidade do caldo das variedades pesquisadas. Verificou-se que as diferenças em qualidade medidas por brix dos tratamentos foram influenciadas diretamente pelos diferentes níveis de potássio, uma vez que estava mais disponível para a planta e participa de diversas funções indispensáveis na cana-de-açúcar, a exemplo do processo fotossintético, determinante na síntese de açúcares. 
Tabela 3. Médias dos valores de brix e umidade interagindo cada nível de adubação dentro dos períodos de supressões ( "DIAC") e variedades.

\begin{tabular}{|c|c|c|c|c|c|c|c|}
\hline VAR & DIAC & NÍVEIS & & & & & \\
\hline & & 1 & 2 & 3 & 4 & 5 & 6 \\
\hline \multicolumn{8}{|c|}{ BRIX(\%) } \\
\hline \multirow{3}{*}{ SP80-1816 } & 165 & $21,89 a$ & $21,85 a$ & $22,58 a$ & $21,97 a$ & $23,36 a$ & $22,55 a$ \\
\hline & 195 & $21,41 a$ & $23,35 a$ & $22,38 b$ & $23,25 a$ & $21,87 b$ & $23,06 a$ \\
\hline & 225 & $22,79 a$ & $23,57 a$ & $24,03 a$ & $24,39 a$ & $24,07 a$ & $23,85 a$ \\
\hline \multirow{3}{*}{ RB85-5453 } & 165 & $24,74 a$ & $25,36 a$ & $25,22 a$ & $25,24 a$ & $25,68 a$ & $26,01 \mathrm{a}$ \\
\hline & 195 & $24,84 a$ & $25,33 a$ & $24,97 a$ & $24,57 a$ & $25,45 a$ & $25,10 a$ \\
\hline & 225 & $23,81 a$ & $25,08 a$ & $25,71 a$ & $25,55 a$ & $26,25 a$ & $25,87 a$ \\
\hline \multicolumn{8}{|c|}{ UMIDADE $(\%)$} \\
\hline \multirow{3}{*}{ SP80-1816 } & 165 & $68,28 a$ & $70,00 a$ & $68,56 a$ & $69,10 a$ & $67,96 a$ & $68,38 a$ \\
\hline & 195 & $68,10 a$ & $67,55 a$ & $68,81 a$ & $67,68 a$ & $69,20 a$ & $67,65 a$ \\
\hline & 225 & $67,41 a$ & $67,66 a$ & $67,18 a$ & $66,26 a$ & $66,73 a$ & $67,30 a$ \\
\hline \multirow{3}{*}{ RB85-5453 } & 165 & $64,70 a$ & $65,46 a$ & $65,45 a$ & $65,63 a$ & $65,06 a$ & $64,63 a$ \\
\hline & 195 & $64,36 a$ & $64,88 a$ & $65,28 a$ & $66,00 a$ & $65,35 a$ & $65,30 a$ \\
\hline & 225 & $64,98 a$ & $65,81 a$ & $64,61 a$ & $64,68 a$ & $63,80 a$ & $64,01 a$ \\
\hline
\end{tabular}

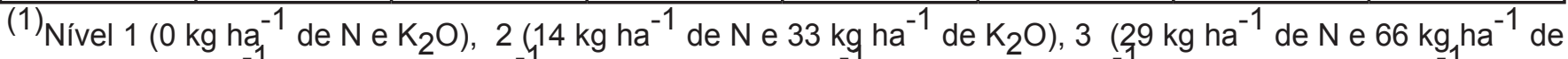
$\left.\mathrm{K}_{2} \mathrm{O}\right), 4\left(43 \mathrm{~kg} \mathrm{ha}^{-1}\right.$ de N e $\left.100 \mathrm{~kg} \mathrm{ha}^{-1} \mathrm{de} \mathrm{K}_{2} \mathrm{O}\right), 5\left(57 \mathrm{~kg} \mathrm{ha}^{-1}\right.$ de Ne $133 \mathrm{~kg} \mathrm{ha}^{-1}$ de $\left.\mathrm{K}_{2} \mathrm{O}\right), 6\left(71 \mathrm{~kg} \mathrm{ha}^{-1} \mathrm{de} \mathrm{Ne}^{-}\right.$ $166 \mathrm{~kg} \mathrm{ha}^{-1}$ de $\mathrm{K}_{2} \mathrm{O}$ ). (2)Médias dentro de cada atributo seguidas por letras minúsculas distintas na linha diferem entre si estatisticamente pelo teste de Scott Knott a $5 \%$ de probabilidade.

Sua ação está intimamente associada à natureza catalítica na formação de carboidratos e no desdobramento e translocação do amido.

Dantas Neto et al. (2006), estudando diferentes lâminas totais de irrigação e doses de adubação nitrogênio + potássio, não registraram diferença na qualidade tecnológica da variedade SP 79-1011, sugerindo que a adubação com nitrogênio não interfere na qualidade tecnológica da cana-de-açúcar, mas influencia no primeiro estádio fenológico, o vegetativo, assim corroborando com os obtidos por Costa et al. (2003), que verificaram que a adubação nitrogenada (100 kg ha-1) não afetou a qualidade tecnológica da cana-de-açúcar.

Prado e Pancelli (2006) obtiveram resultados na primeira soqueira, e observaram que não houve efeito significativo dos diferentes tratamentos de adubação nitrogenada na qualidade tecnológica dos colmos da soqueira de cana. Moura et al. (2005) encontraram valores próximos de Brix para diferentes doses de adubação de cobertura de nitrogênio com irrigação, com valor médio de $19,76^{\circ}$ Brix de variedade não relatada.

Na tabela 4 têm-se os valores médios AR, ATR e ART, que apresentaram valores médios de 0,598\%, 172,389kg ATR t-1 e 18,98 \% ART (tabela 1 ), respec- tivamente. No parâmetro AR observou-se interação em todos os tratamentos com a variedade SP80-1816 superando os valores na maioria dos tratamentos. Esses valores estão dentro dos aceitáveis na produção sucroalcooleira, pois esse parâmetro em grandes quantidades se encontra na condição de prejuízos na produção de álcool e principalmente de açúcar.

Destacou-se na tabela 4 valor máximo de $0,8 \%$ AR no DIAC 165 da variedade SP80-1816 no nível 2 e mínimo na variedade RB85-5453 aos 225 DIAC no nível 4 com $0,35 \%$ AR. Estes valores dão indícios que um período vegetativo de boa disponibilidade hídrica e nutritiva para a cultura da cana na região Nortemineira desempenha bons rendimentos de sacarose acumulada no colmo da cana e boa produtividade que atenda ao processamento industrial de açúcar e álcool.

Prado e Pancelli (2006), analisando diferentes adubações com nitrogênio, encontraram valores de $0,65 \%$ AR com adubação de $200 \mathrm{~kg}$ ha-1, e 0,83\% AR com $0 \mathrm{~kg}$ ha-1, evidenciaram que os diferentes tratamentos não influenciaram esta qualidade tecnológica da cana. Esses resultados corroboram os encontrados neste trabalho, demonstrando que os diferentes níveis de adubação não foram influentes na \%AR (tabela 4).

Tabela 4. Médias dos valores de porcentagens de Açúcares redutores (AR), Açúcares teórico recuperáveis (ATR) e litros álcool esperado por tonelada de cana (LITRS ÁLCOOL) do caldo no desdobramento do período de irrigação e precipitação (DIAC) com variedades dentro de cada nível de adubação. 
Revista Agroambiental - Agosto/2011

\begin{tabular}{|c|c|c|c|c|c|c|c|}
\hline & & \multicolumn{2}{|c|}{$A R(\%)$} & \multicolumn{2}{|c|}{ ATR(\%) } & \multicolumn{2}{|c|}{ ART(\%) } \\
\hline NÍVEIS & DIAC & SP80-1816 & RB85-5453 & SP80-1816 & RB85-5453 & SP80-1816 & RB85-5453 \\
\hline \multirow{3}{*}{1} & 165 & $0,55 \mathrm{aC}$ & $0,59 a A$ & $166,92 \mathrm{bA}$ & $181,23 a A$ & $18.44 \mathrm{bA}$ & $20.02 \mathrm{aA}$ \\
\hline & 195 & $0,74 a \mathrm{~A}$ & $0,55 \mathrm{bB}$ & $159,74 \mathrm{bA}$ & $180,82 \mathrm{aA}$ & $17.65 \mathrm{bA}$ & $19.98 \mathrm{aA}$ \\
\hline & 225 & $0,68 \mathrm{aB}$ & $0,62 \mathrm{bA}$ & $166,35 \mathrm{bA}$ & $180,11 \mathrm{aA}$ & 18.38aA & $19.90 \mathrm{aA}$ \\
\hline \multirow{3}{*}{2} & 165 & $0,80 a A$ & $0,62 \mathrm{bA}$ & $147,86 \mathrm{bB}$ & $181,06 a \mathrm{~A}$ & $15.78 \mathrm{bB}$ & $20.00 \mathrm{aA}$ \\
\hline & 195 & $0,73 \mathrm{aB}$ & $0,53 \mathrm{bB}$ & $162,21 \mathrm{bA}$ & $181,47 a A$ & $17.48 \mathrm{bA}$ & $20.03 \mathrm{aA}$ \\
\hline & 225 & $0,67 \mathrm{aB}$ & $0,55 \mathrm{bB}$ & $166,60 \mathrm{aA}$ & $175,26 a A$ & 18.04aA & $19.36 \mathrm{aA}$ \\
\hline \multirow{3}{*}{3} & 165 & $0,60 \mathrm{aA}$ & $0,56 a A$ & $161,67 \mathrm{bB}$ & $181,96 a A$ & $17.68 \mathrm{bB}$ & $20.10 \mathrm{aA}$ \\
\hline & 195 & $0,72 \mathrm{aC}$ & $0,53 \mathrm{bA}$ & $161,53 \mathrm{bB}$ & $181,34 a A$ & $17.66 \mathrm{bB}$ & $20.04 \mathrm{aA}$ \\
\hline & 225 & $0,52 a A$ & $0,46 \mathrm{bB}$ & $175,35 a A$ & $186,44 a \mathrm{~A}$ & 19.37aA & $20.60 \mathrm{aA}$ \\
\hline \multirow{3}{*}{4} & 165 & $0,67 a A$ & $0,59 \mathrm{bA}$ & $151,39 \mathrm{bB}$ & $179,47 a A$ & $17.04 \mathrm{bB}$ & $19.83 \mathrm{aA}$ \\
\hline & 195 & $0,67 \mathrm{aB}$ & $0,44 \mathrm{bB}$ & $160,16 \mathrm{bB}$ & $178,98 a A$ & $17.69 \mathrm{bB}$ & $19.77 \mathrm{aA}$ \\
\hline & 225 & $0,59 a C$ & $0,35 \mathrm{bC}$ & $173,57 \mathrm{bA}$ & $186,53 a A$ & 19.18aA & $20.61 \mathrm{aA}$ \\
\hline \multirow{3}{*}{5} & 165 & $0,68 a A$ & $0,60 \mathrm{bA}$ & $163,18 \mathrm{bA}$ & $181,88 a A$ & $17.71 \mathrm{bA}$ & $20.09 \mathrm{aA}$ \\
\hline & 195 & $0,65 a A$ & $0,56 \mathrm{bB}$ & $153,76 \mathrm{bA}$ & $179,65 a \mathrm{~A}$ & $17.06 \mathrm{bA}$ & $19.85 \mathrm{aA}$ \\
\hline & 225 & $0,65 a A$ & $0,54 \mathrm{bB}$ & $168,86 \mathrm{bA}$ & $183,44 a A$ & $18.65 \mathrm{bA}$ & 20.27 aA \\
\hline \multirow{3}{*}{6} & 165 & $0,56 a \mathrm{~B}$ & $0,49 \mathrm{bB}$ & $156,87 \mathrm{bA}$ & $189,25 a \mathrm{~A}$ & $17.33 \mathrm{bA}$ & $20.91 \mathrm{aA}$ \\
\hline & 195 & $0,66 a A$ & $0,47 \mathrm{bB}$ & $162,12 \mathrm{bA}$ & $185,46 a A$ & $17.91 \mathrm{bA}$ & $20.49 \mathrm{aA}$ \\
\hline & 225 & $0,69 a A$ & $0,54 \mathrm{bA}$ & $166,59 \mathrm{bA}$ & $186,76 a A$ & $18.40 \mathrm{bA}$ & $20.08 \mathrm{aA}$ \\
\hline
\end{tabular}

(1)Nível 1 (0 kg ha-1 de Ne K2O), 2 (14 kg ha-1 de N e 33 kg ha-1 de K2O), 3 (29 kg ha-1 de N e 66 kg ha-1 de K2O), 4 (43 kg ha-1 de N e 100 kg ha-1 de K2O), 5 (57 kg ha-1 de N e 133 kg ha-1 de K2O), 6 (71 kg ha-1 de N e $166 \mathrm{~kg}$ ha-1 de K2O). (2)Médias dentro de cada atributo seguidas por letras minúsculas distintas na linha diferem entre si estatisticamente pelo teste de Scott Knott a $5 \%$ de probabilidade. (3)Médias dentro de cada nível seguidas por letras maiúsculas distintas na coluna diferem entre si estatisticamente pelo teste de Scott Knott a $5 \%$ de probabilidade

Dos atributos analisados, o açúcar total recuperável (ATR) é muito importante tanto para indústria quanto para os produtores. Pois em função dele é que as unidades industriais elaboram o preço pago aos produtores, seguindo uma metodologia descrita pela (CONSECANA, 2006).

Observando o parâmetro ATR, verificou-se que a variedade RB85-5453 apresentou melhor resultado, tendo possibilidade de atingir teores superiores a 189,25 $\mathrm{kg}$ de ATR t-1. Nos tratamentos de DIAC houve diferenças significativas $(p<0,05)$ apenas aos 165 DIAC nos níveis 2, 3 e 4, indicando que maior disponibilidade hídrica favorece o rendimento teórico de açúcar.

$\mathrm{Na}$ variável ART a variedade $\mathrm{SP}$ obteve um desempenho melhor com valores menores em porcen- tagem, atingindo os $17,04 \%$ ART no manejo 4 aos 165 DIAP, valor máximo de $19,37 \%$ ART no manejo 3 aos 225 DIAP este não diferiu dos valores encontrados na variedade SP. Isto é evidente uma vez que os valores de $\%$ AR na variedade SP foram maiores, tendo uma tendência de maior recuperação na variedade RB de menor $\%$ AR. Os manejos de adubação não diferiram nos valores de \%ART (tabela 2), não influenciando seu resultado final.

Todos os índices discutidos neste trabalho são usados como base de cálculo para se determinar a quantidade de açúcares totais recuperáveis, expressos em kg de ATR t-1 de cana. Pelos resultados desta pesquisa comprova-se que a qualidade da matéria-prima pode ser melhorada com a irrigação.

Tabela 5 Médias dos valores de porcentagens de açúcares redutores (AR), açúcares teórico recuperáveis (ATR) do caldo e litros álcool esperado por tonelada de cana (LITRS ALCOOL), interagindo cada nível de adubação dentro dos períodos ( Dias de irrigação mais precipitação após corte "DIAC") e variedades 


\begin{tabular}{|c|c|c|c|c|c|c|c|}
\hline VAR & DIAC & NÍVEIS & & & & & \\
\hline & & 1 & 2 & 3 & 4 & 5 & 6 \\
\hline \multicolumn{8}{|c|}{ AR (\%) } \\
\hline \multirow{3}{*}{ SP80-1816 } & 165 & $0,55 \mathrm{c}$ & $0,80 \mathrm{a}$ & $0,60 \mathrm{c}$ & $0,67 \mathrm{~b}$ & $0,68 \mathrm{~b}$ & $0,56 c$ \\
\hline & 195 & $0,74 a$ & $0,73 \mathrm{a}$ & $0,72 \mathrm{a}$ & 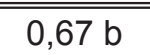 & $0,65 \mathrm{~b}$ & "0,66b \\
\hline & 225 & $0,68 \mathrm{a}$ & $0,67 \mathrm{a}$ & $0,52 \mathrm{c}$ & $0,59 \mathrm{~b}$ & $0,65 a$ & "0,69a \\
\hline \multirow{3}{*}{ RB85-5453 } & 165 & $0,59 a$ & $0,62 \mathrm{a}$ & $0,56 a$ & $0,59 a$ & $0,60 \mathrm{a}$ & $0,49 b$ \\
\hline & 195 & $0,55 a$ & $0,53 \mathrm{a}$ & $0,53 a$ & $0,44 \mathrm{~b}$ & $0,56 \mathrm{a}$ & $0,47 b$ \\
\hline & 225 & $0,62 \mathrm{a}$ & $0,55 \mathrm{~b}$ & $0,46 \mathrm{c}$ & $0,35 \mathrm{~d}$ & $0,54 \mathrm{c}$ & $0,54 \mathrm{c}$ \\
\hline \multicolumn{8}{|c|}{ ATR $\left(\mathrm{kg} \mathrm{t}^{-1}\right)$} \\
\hline \multirow{3}{*}{ SP80-1816 } & 165 & $166,92 a$ & $147,86 b$ & $161,67 a$ & $151,39 b$ & $161,67 a$ & $156,87 b$ \\
\hline & 195 & $159,74 a$ & $162,21 \mathrm{a}$ & $161,53 a$ & $160,16 a$ & $153,76 a$ & $162,12 \mathrm{a}$ \\
\hline & 225 & $166,35 a$ & $166,60 a$ & $175,35 a$ & $173,57 a$ & $168,86 a$ & $166,59 a$ \\
\hline \multirow{3}{*}{ RB85-5453 } & 165 & $181,23 a$ & $181,06 a$ & $181,96 a$ & $179,47 a$ & $181,88 a$ & $189,25 a$ \\
\hline & 195 & $180,82 a$ & $181,47 a$ & $181,34 a$ & $178,98 a$ & $179,65 a$ & $\overline{185,46 a}$ \\
\hline & 225 & $180,11 a$ & $175,26 a$ & $186,44 a$ & $186,53 a$ & $183,44 a$ & $\overline{186,76 a}$ \\
\hline \multicolumn{8}{|c|}{ ART (\%) } \\
\hline \multirow{3}{*}{ SP80-1816 } & 165 & $18.44 \mathrm{a}$ & $15.78 \mathrm{~b}$ & $17.68 \mathrm{a}$ & $17.04 \mathrm{a}$ & $17.71 \mathrm{a}$ & $17.33 \mathrm{a}$ \\
\hline & 195 & $17.65 \mathrm{a}$ & $17.48 \mathrm{a}$ & $17.66 \mathrm{a}$ & $17.69 \mathrm{a}$ & $17.06 \mathrm{a}$ & $17.91 \mathrm{a}$ \\
\hline & 225 & $18.38 \mathrm{a}$ & $18.04 \mathrm{a}$ & $19.37 \mathrm{a}$ & $19.18 \mathrm{a}$ & $18.65 \mathrm{a}$ & $18.40 \mathrm{a}$ \\
\hline \multirow{3}{*}{ RB85-5453 } & 165 & $20.02 \mathrm{a}$ & $20.00 \mathrm{a}$ & $20.10 \mathrm{a}$ & $19.83 \mathrm{a}$ & $20.09 \mathrm{a}$ & $20.91 \mathrm{a}$ \\
\hline & 195 & $19.98 \mathrm{a}$ & $20.03 a$ & $20.04 \mathrm{a}$ & $19.77 \mathrm{a}$ & $19.85 \mathrm{a}$ & $20.49 a$ \\
\hline & 225 & $19.90 \mathrm{a}$ & $19.36 \mathrm{a}$ & $20.60 \mathrm{a}$ & $20.61 \mathrm{a}$ & $20.27 \mathrm{a}$ & $20.08 \mathrm{a}$ \\
\hline
\end{tabular}

\section{CONCLUSÃO}

1. O período de 225 DIAC foi o melhor em desempenho, porém tendo valores próximos em qualidade tecnológica nas demais supressões.

2. O nível 6 de adubação obteve melhor desempenho nas condições de realização deste trabalho.

3. A variedade RB85-5453 teve maior desempenho em qualidade tecnológica mostrando-se adequada a colheita mais cedo na região.

4. As variedades RB85-5453 e SP80-1816 são promissoras para região Nortemineira, tendo boa adaptação às condições edafoclimáticas, porém a variedade RB85-5453 é a mais indicada para a região, nas condições de realização deste trabalho, assim atendendo às exigências de produção sucroalcooleira no seu terceiro ciclo de produtividade.

\section{REFERÊNCIAS BIBLIOGRÁFICAS}

[1] BERNARDO, S. Manual de irrigação. 7. ed. Viçosa: Impressa Universitária, 2005. 611 p.

[2] COMISSÃO DE FERTILIDADE DE SOLO DO ESTADO DE MINAS GERAIS (CFSEMG). Recomendações para o uso de corretivos e fertilizantes em Minas Gerais; $5^{a}$ aproximação. Lavras: CFSEMG, 1999. 359p.
[3] CÉSAR, M.A.A.; DELGADO, A.A.; CAMARGO, A.P.de; BISSOLI, B.M.A.; SILVA, F.C.da. Capacidade de fosfatos naturais e artificiais em elevar o teor de fósforo no caldo de cana-de-açúcar (cana-planta), visando o processo industrial. STAB, v.6, p.32-38, 1987.

[4] CONSECANA. . Conselho dos Produtores de Cana-de-Açúcar, Açúcar e Álcool do Estado de São Paulo; Manual de Instruções , $5^{\circ}$ Edição, PiracicabaSP, 112 p, 2006.

[5] COSTA, M.C.G.; VITTI, G.C.; CANTARELLA, H. Volatilização de N-NH3 de fontes nitrogenadas em cana-deaçúcar colhida sem despalha a fogo. Revista Brasileira de Ciência do Solo, Viçosa, v.27, n.4, p.631-637, 2003.

[6] DANTAS NETO, J. et al. Resposta de cana-de-açúcar, primeira soca, a níveis de irrigação e adubação de cobertura. Revista Brasileira de Engenharia Agrícola e Ambiental, Campina Grande, v. 10, n. 2, p. 283-288, 2006.

[7] DELGADO, A.A.; CÉSAR, M.A.A. Elementos de tecnologia e engenharia do açúcar de cana. Sertãozinho: Zanini, 1977. 1041p. 
[8] DOOREMBOS, J.; KASSAM, A. H. Yield response to water. Rome: FAO, 1979. 193 p. Irrigation and Drainage Paper, 33.

[9] EMBRAPA. Centro Nacional de Pesquisa de Solos. Sistema brasileiro de classificação de solos. Brasília: SPI, 1999. 412 p.

[10] HORII, J. A qualidade da matéria-prima, na visão agrícola. Visão Agrícola, v.1, n.1, 2004, p.91-93.

[11] LOPES, C.; PARAZZI, C. Introdução à tecnologia de produção de açúcar. São Carlos: UFSCar, 1992. 151p.

[12] MELO, F. A. D. et al. Parâmetros Tecnológico da cana-de-açúcar em diferentes fundos agrícolas da região Norte do Estado do Pernambuco. In: CONGRESSO NACIONAL DA STAB, 7., 1998. Londrina. Anais... Piracicaba: STAB, 1999. p. 198-202.

[13] MOURA-FILHO, G. et al. GD-CANA-programa cálculo de graus-dia para a cultura da cana-de-açúcar. In: CONGRESSO BRASILEIRO DE AGROMETEOROLOGIA, 14., 2005, Campinas, SP. Anais... Campinas: Sociedade Brasileira de Agrometeorologia, 2005. 1 CDROM.

[14] PARANHOS, S. Cana-de-açúcar cultivo e utilização. Campinas: Fundação Cargill, 1987. 356p.

[15] PRADO, R. de M.; PANCELLI, M. A. Nutrição em soqueira e a qualidade tecnológica da cana-de-açúcar. STAB: Açúcar, Álcool e Subprodutos, Piracicaba, v. 25, n. 2, p. 60-63, 2006.

[16] WIEDENFELD, R. P. Effects of irrigation and N fertilizer application on sugar cane yield and quality. Field Crop Research, Amsterdam, v. 43, p. 101-108, 1995.

[17] WIEDENFELD, R. P. Water stress during different sugarcane growth periods on yield and response to $\mathbf{N}$ fertilizer. Agricultural Water Management., Elsevier, v.43 p.173-182, 2000. 